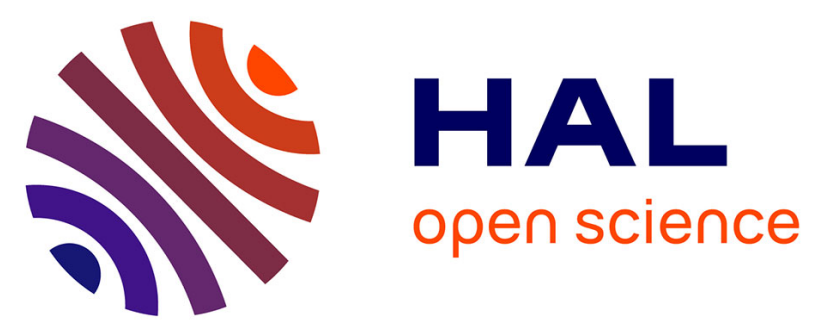

\title{
TRACKING PERFORMANCE ACHIEVEMENT FOR CONTINUOUS-TIME DELAYED LINEAR SYSTEMS SUBJECT TO ACTUATOR SATURATION AND OUTPUT DISTURBANCES
}

Adel Mahjoub, Vincent van Assche, Fouad Giri, Fatima Zara Chaoui

\section{To cite this version:}

Adel Mahjoub, Vincent van Assche, Fouad Giri, Fatima Zara Chaoui. TRACKING PERFORMANCE ACHIEVEMENT FOR CONTINUOUS-TIME DELAYED LINEAR SYSTEMS SUBJECT TO ACTUATOR SATURATION AND OUTPUT DISTURBANCES. Asian Journal of Control, 2015, 17 (4), pp.PP1-7. 10.1002/asjc.990 . hal-01102060

\section{HAL Id: hal-01102060 https://hal.science/hal-01102060}

Submitted on 12 Jan 2015

HAL is a multi-disciplinary open access archive for the deposit and dissemination of scientific research documents, whether they are published or not. The documents may come from teaching and research institutions in France or abroad, or from public or private research centers.
L'archive ouverte pluridisciplinaire HAL, est destinée au dépôt et à la diffusion de documents scientifiques de niveau recherche, publiés ou non, émanant des établissements d'enseignement et de recherche français ou étrangers, des laboratoires publics ou privés. 


\title{
TRACKING PERFORMANCE ACHIEVEMENT FOR CONTINUOUS-TIME DELAYED LINEAR SYSTEMS SUBJECT TO ACTUATOR SATURATION AND OUTPUT DISTURBANCES
}

\author{
Adel Mahjoub, Vincent Van Assche, Fouad Giri, and Fatima Z. Chaoui
}

\begin{abstract}
Controlling continuous-time input-delayed nonminimum-phase linear systems is addressed in the presence of actuator saturation and output-disturbances. Focusing on output-reference tracking, the control design is dealt with in the pseudo-polynomials ring. A quite appealing $L_{2}$-tracking performance is shown to be achievable in the presence of arbitrary inputs i.e. the output reference and the output disturbance. The performance is formulated in terms of a well defined output-reference mismatch error (ORME), depending on the inputs' rate and their compatibility with the actuator saturation constraint.
\end{abstract}

Key Words: Linear systems, input delay, actuator saturation, tracking performances.

\section{INTRODUCTION}

Controlling linear systems via saturating actuators has been studied extensively, especially over the last 15 years. The solutions proposed so far have been developed following two main paths, respectively referred to as anti-windup compensator (AWC) synthesis and direct control design (DCD). In the first approach, a predefined controller ensuring satisfactory control performances in the absence of actuator saturation is supposed to be available. Then, an additional compensator is designed to minimize the adverse effect of actuator saturation on closed-loop performances. The DCD problem is one where the input constraint is accounted for at the controller design stage. In addition to actuator saturation, physical systems are also subject to (less or more significant) dead-times and several approaches have been proposed in past years to solve various control problems involving system delays $[1,2]$. The point is that relatively few works have dealt with the problem of controlling delayed systems through saturating actuators. In [3,4] the AWC is designed to ensure $L_{2}$ stability of the operator relating the control saturation error $\widetilde{u}=u-\operatorname{sat}(u)$ (the difference between the control input $u$ generated in the closed-loop without input saturation and the same signal passed through the saturation nonlinearity) to $\widetilde{z}=z-\bar{z}$ (the error between the control system performance outputs with and without input saturation). However, the class of inputs (references, disturbances) for which the condition $\widetilde{u} \in L_{2}$ holds is not explicitly defined. Furthermore, it is not clear how the constrained closed-loop system behaves when the inputs are not constraint-compatible so that $\widetilde{u} \notin L_{2}$. The problem of controlling delayed systems through

A. Mahjoub is with the University of Sfax, Sfax, Tunisia.

V. Van Assche and F. Giri (corresponding author, e-mail: fouadgiri@yahoo.fr) are with the University of Caen Basse-Normandie, GREYC Lab, UMR CNRS, 14032 Caen, France.

F. Z. Chaoui is with ENSET, University of Rabat Souissi, Rabat, Morocco. saturating actuators has also been dealt with using the DCD approach and a number of results have been obtained e.g. global stabilization [5], asymptotic stabilization with optimized domain of attraction $[6,7]$. Extensions to multiple and distributed actuator delays and saturations were investigated in [8]. A common point to all former works is that only system stabilization results have been achieved, assuming all inputs to be null (reference signals and output disturbances). In this paper, the focus is placed on asymptotic tracking of arbitrary-shape reference signals when input-delayed nonminimum-phase stable systems are controlled via saturating actuators and their outputs are subject to disturbances. The control design is presently dealt with in the ring of a pseudopolynomial making use of the (finite-spectrum) pole-placement technique $[9,10]$. A saturating controller is thus developed and analyzed using tools from the pseudo-polynomial algebra and the input-output stability [11]. It is formally proved that, in addition to closed-loop global stability, the controller enjoys a quite interesting $L_{2}$-tracking performance. Specifically, it is formally established that the (nonlinear) performance mapping, $O R M E \rightarrow e_{y}$ is $L_{2}$-stable where $e_{y}$ denotes the tracking error and ORME, the output-reference mismatch error, is a well defined quantity depending on the rate of the reference signal and its compatibility with the constraint induced by the actuator saturation. Note that, in practical applications, reference compatibility may be difficult to check or even lost due to model uncertainties. Then, it is of practical interest to analyze the tracking capability of the controller facing constraint-incompatible reference signals of arbitrary shape. This issue has not previously been investigated in the context of input-constrained dead-time systems [3]. It turns out that the $L_{2}$-tracking performances established in the present paper constitute original results. The present control design and analysis approach, which is not very usual in constrained deadtime systems, finds some roots in [12] where the focus was made on discrete-time systems. The present paper is an improved and a much more complete version of the conference paper [13]. It is organized as follows: Section II is devoted to formulating the control problem; the controller is designed in Section III and 
analyzed in Section IV; the corresponding tracking performances are illustrated by simulation in Section V.

\section{CONTROL PROBLEM STATEMENT}

We are interested in controlling input-delayed linear systems of the form:

$$
\begin{aligned}
& \hat{y}(s)=\frac{B(s) e^{-s \tau}}{A(s)} \hat{u}(s)+\hat{w}(s) \\
& A(s)=s^{n}+a_{n-1} s^{n-1}+\ldots+a_{1} s+a_{0}, \\
& B(s)=b_{n-1} s^{n-1}+\ldots+b_{1} s+b_{0}
\end{aligned}
$$

in presence of the input constraint:

$$
|u(t)| \leq u_{M}
$$

where $(u(t), y(t))$ are the system input and output and $(\hat{u}(s), \hat{y}(s))$ their Laplace transforms; similarly $w(t)$ represents the disturbance; $u_{M}>0$ the maximal control amplitude; the integer $n$ is the system order and the real numbers $\left(a_{i}, b_{i}\right)$ its parameters. It is supposed that $\mathrm{A}(s)$ is Hurwitz and $(s A(s), B(s))$ are coprime and $b_{0} \neq 0$. The aim of the present study is to develop a controller that makes the tracking error,

$$
e_{y}=y-y^{*}
$$

as small as possible, where $y^{*}$ denotes any bounded reference input. The point is that the nonminimum phase nature of the system makes perfect tracking (e.g. $e_{y} \in L_{2}$ ) unachievable in presence of arbitraryshape reference signals. The class of (constant) system inputs for which perfect matching is achievable will be called constraintcompatible. To formally define this notion, introduce the following signal:

$$
\begin{aligned}
u^{*}(t) & =\frac{A(0)}{B(0)}\left(y^{*}(t+\tau)-w(t+\tau)\right) \\
& =\frac{a_{0}}{b_{0}}\left(y^{*}(t+\tau)-w(t+\tau)\right)
\end{aligned}
$$

It is readily checked that, if the control system inputs $\left(y^{*}, w\right)$ are constant, using (1) one can obtain:

$$
u(t)=u^{*}(t), \forall t \Rightarrow y(t)=y^{*}(t), \forall t
$$

That is, the signal $u^{*}(t)$ defines the ideal control trajectory for the achievement of perfect tracking, in the absence of input constraint, when the control system inputs $\left(y^{*}, w\right)$ are constant. For this achievement to be preserved in presence of the input constraint, it is necessary that:

$$
\left|u^{*}(t)\right| \leq u_{M}, \text { or equivalently, } u^{*}-\operatorname{sat}\left(u^{*}\right)=0
$$

where sat(.) denotes the saturation function defined by:

$$
\operatorname{sat}(z)=\min \left(u_{M},|z|\right) \operatorname{sgn}(z), z \in \boldsymbol{R}
$$

In the light of the above discussion, it turns out that the achievement of perfect output-reference tracking, for nonminimum phase systems, is possible in presence of constant constraintcompatible inputs, i.e., those satisfying $\left[\dot{y}^{*} \dot{w} u^{*}-\operatorname{sat}\left(u^{*}\right)\right]=$ $\left[\begin{array}{lll}0 & 0 & 0\end{array}\right]$. With arbitrary-shape input signals, the tracking quality is expected to be dependent on how much do the system inputs deviate from the ideal shape. For this reason, the vector $\left[\dot{y}^{*} \dot{w} u^{*}-\operatorname{sat}\left(u^{*}\right)\right]^{T}$ will be referred to as the output-reference mismatch error (ORME). The smaller (the norm of) this error vector, the better the tracking quality is expected to be. This objective is presently formalized by requiring the mapping,

$$
\left[\dot{y}^{*} \quad \dot{w} \quad u^{*}-\operatorname{sat}\left(u^{*}\right)\right]^{T} \rightarrow e_{y}
$$

to be $L_{2}$-stable. Accordingly [11], it is required that a pair of positive real constants $(\alpha, \beta)$ exists such that one has, for all bounded system inputs $\left(y^{*}, w\right)$ and for any real $T>0$ :

$$
\begin{aligned}
\left(\int_{0}^{T}\left|e_{y}(t)\right|^{2} d t\right)^{1 / 2} & \leq \alpha\left(\int_{0}^{T} \dot{y}^{*}(t)^{2} d t\right)^{1 / 2}+\alpha\left(\int_{0}^{T} \dot{w}(t)^{2} d t\right)^{1 / 2} \\
& +\alpha\left(\int_{0}^{T}\left(u^{*}(t)-\operatorname{sat}\left(u^{*}(t)\right)\right)^{2} d t\right)^{1 / 2}+\beta
\end{aligned}
$$

with || || denoting the Euclidean norm. Each of the two statements (6a) and (6b) will be referred to as the $L_{2}$-tracking performance. This entails the following property:

$$
\begin{gathered}
\left\|e_{y}\right\|_{2} \leq \alpha\left(\left\|u^{*}-\operatorname{sat}\left(u^{*}\right)\right\|_{2}+\left\|\dot{y}^{*}\right\|_{2}+\|\dot{w}\|_{2}\right), \\
\text { whenever } \dot{y}^{*}, \dot{w}, \quad\left(u^{*}-\operatorname{sat}\left(u^{*}\right)\right) \in L_{2},
\end{gathered}
$$

Of course, in the general case of arbitrary-shape and/or not constraint-compatible inputs, one has $\dot{y}^{*} \notin L_{2}$ or $\dot{w} \notin L_{2}$ or $\left(u^{*}-\right.$ $\left.\operatorname{sat}\left(u^{*}\right)\right) \notin L_{2}$. Then, (6b) can not be expressed using the $L_{2}$-norm. To deal with this more general situation a convenient "norm" must be used. To this end, it follows dividing both sides of (6b) by $T^{1 / 2}$ and letting $T \rightarrow \infty$ :

$$
\begin{gathered}
\limsup _{T \rightarrow \infty}\left(\frac{1}{T} \int_{0}^{T}\left|e_{y}(t)\right|^{2} d t\right)^{1 / 2} \leq \alpha \limsup _{T \rightarrow \infty}\left(\frac{1}{T} \int_{0}^{T}\left|\dot{y}^{*}(t)\right|^{2} d t\right)^{1 / 2} \\
+\alpha \underset{T \rightarrow \infty}{\limsup }\left(\frac{1}{T} \int_{0}^{T}|\dot{w}(t)|^{2} d t\right)^{1 / 2} \\
+\alpha \limsup _{T \rightarrow \infty}\left(\frac{1}{T} \int_{0}^{T} \mid\left(u^{*}-\left.\operatorname{sat}\left(u^{*}\right)\right|^{2} d t\right)^{1 / 2}\right.
\end{gathered}
$$

This motivates the notation, $\quad\|x\|_{2 a} \stackrel{\text { def }}{=} \limsup _{T \rightarrow \infty}$ $\left(\frac{1}{T} \int_{0}^{T}|x(t)|^{2} d t\right)^{1 / 2}$. Accordingly, the $L_{2}$-tracking performance (6b) entails the following property:

$$
\begin{aligned}
& \left\|e_{y}\right\|_{2 a} \leq \alpha\left(\left\|u^{*}-\operatorname{sat}\left(u^{*}\right)\right\|_{2 a}+\left\|\dot{y}^{*}\right\|_{2 a}+\|\dot{w}\|_{2 a}\right) \\
& \quad \text { if } \dot{y}^{*}, \quad \dot{w}, \quad\left(u^{*}-\operatorname{sat}\left(u^{*}\right)\right) \in L_{\infty}
\end{aligned}
$$

In summary, the control problem at hand consists in designing, for the stable system (1) operating under the constraint (3) and the disturbance input $w$, a controller that guarantees the $L_{2}$-stability of the performance operator (6a) which entails the two tracking properties (7a) and (7c). 


\section{CONTROLLER DESIGN}

The control design is an extension of the input-constrained case of the finite-spectrum assignment (FSA) method [10,12]. Just as in the standard pole assignment method, the starting point is an arbitrary choice, by the designer, of a pair of Hurwitz polynomials of the form:

$$
\begin{aligned}
& C(s)=s^{n}+c_{n-1} s^{n-1}+\ldots+c_{1} s+c_{0}, \\
& \Lambda(s)=s^{n}+\lambda_{n-1} s^{n-1}+\ldots+\lambda_{1} s+\lambda_{0},
\end{aligned}
$$

As $s A(\mathrm{~s})$ and $B(s)$ are coprime on $\mathbf{R}[s]$ (ring of polynomials with real coefficients), they are also coprime on $\mathbf{E}$ the ring of pseudo-polynomials $[9,10]$. Then, there exists a pair of pseudopolynomials $R^{\prime}(\mathrm{s})$ and $P^{\prime}(\mathrm{s})$ satisfying the Bezout equation:

$$
R^{\prime}(s) s A(s)+P^{\prime}(s) B(s) e^{-s \tau}=C(s) \Lambda(s)
$$

Following [10,12], one divides $P^{\prime}(s)$ by the monic polynomial $s A(s)$ and gets $P^{\prime}(s)=Q(s) s A(s)+P(s)$ with $\operatorname{deg} P(s) \leq n$. Then, letting $R(s)=R^{\prime}(s)+Q(s) B(s) e^{-s \tau}$, (9) rewrites:

$$
R(s) s A(s)+P(s) B(s) e^{-s \tau}=C(s) \Lambda(s)
$$

As $\operatorname{deg}\left(P(s) B(s) e^{-s \tau}\right) \leq 2 n-1$, it follows that $\operatorname{deg}(R(s)$ $s A(s))=\operatorname{deg}(C(s) \Lambda(s))=2 n$ which in turn implies that $\operatorname{deg}(R(s))=$ $n-1$, because $\operatorname{deg}(s A(s))=n+1$. Furthermore, as $s A(s)$ and $C(s) \Lambda$ $(s)$ are monic, $R(s)$ must in turn be monic (i.e. its higher degree term coefficient equals 1$)$. In the light of the above observations, it is readily seen that $\mathrm{R}(s)$ and $P(s)$ are uniquely expressed as follows:

$$
\begin{aligned}
& R(s)=s^{n-1}+\sum_{i=0}^{n-2} R_{i}\left(e^{-s \tau}\right) s^{i}+R_{-1}(s), \\
& P(s)=\sum_{i=0}^{n} P_{i}\left(e^{-s \tau}\right) s^{i}+P_{-1}(s)
\end{aligned}
$$

where $R_{-1}(s)$ and $P_{-1}(s)$ belong to $\mathbf{G}$, the set of transfer functions of distributed and punctual delay operators. For $i \geq 0, R_{i}$ $\left(e^{-s \tau}\right)$ and $P_{i}\left(e^{-s \tau}\right)$ belong to $\mathbf{R}\left[e^{-s \tau}\right]$, the set of polynomials in $e^{-s \tau}$. Unlike the case of non-delayed systems, the (finite-degree) operators $\mathrm{R}(s)$ and $P(s)$ are presently pseudo-polynomials which, like classical polynomials, are analytical functions of $s$. Now, let us temporarily suppose that the system (1-2) is not subject to the constraint (3). Then, the FSA control method suggests the control law $\hat{u}(s)=-\frac{P(s)}{s R(s)} \hat{e}_{y}(s)$. Clearly, this corresponds to a regulator featuring a unitary-feedback and integral action. For implementation robustness, the above regulator is given in the alternative form $\hat{u}(s)=\frac{\Lambda(s)-s R(s)}{\Lambda(s)} \hat{u}(s)-\frac{P(s)}{\Lambda(s)} \hat{e}_{y}(s)$ where the involved transfer functions are asymptotically stable (because $\Lambda(s)$ is Hurwitz) and causal. As the system input is subject to the constraint (3), the above regulator is modified so that it generates a control action not exceeding the constraint limits. Specifically, the following saturated controller is considered:

$$
\begin{aligned}
& \hat{v}(s)=\frac{\Lambda(s)-s R(s)}{\Lambda(s)} \hat{u}(s)-\frac{P(s)}{\Lambda(s)} \hat{e}_{y}(s) \\
& u(t)=\operatorname{sat}(v(t))=\operatorname{sgn}(v(t)) \min \left(|v(t)|, u_{M}\right)
\end{aligned}
$$

\section{CONTROLLER TRACKING CAPABILITY ANALYSIS}

First, let us show that all signal derivatives are related to the inputs' rate through $L_{2}$-stable operators. Combining (1) and (12a) so that $e_{y}$ is eliminated, one gets the following relation between signals rate:

$$
s \hat{v}(s)=-\frac{C(s)-A(s)}{A(s)}(s \hat{u}(s))+\frac{P(s)}{\Lambda(s)}\left(s \hat{y}^{*}(s)-s \hat{w}(s)\right)
$$

This equation fits the feedback representation of Fig. 1 where:

$$
\hat{\delta}_{1}(s)=\frac{P(s)}{\Lambda(s)}\left(s \hat{y}^{*}(s)-s \hat{w}(s)\right)
$$

and $\psi$ is the mapping: $\dot{v} \rightarrow \dot{u}$. If the operator $\psi$ is shown to lie in some conic sector, absolute stability theorems [11] can be applied to the feedback of Fig. 1, to get bounding on $\dot{u}$ and $\dot{v}$ (see Proposition 1).

Proposition 1 (Signal rate bounding). Let the polynomial $C(s)$ in (10) be chosen such that:

$$
\inf _{0 \leq \omega<+\infty} \operatorname{Re}\left(\frac{C(j \omega)}{A(j \omega)}\right)>0
$$

Then, the feedback of Fig. 1 is $\mathrm{L}_{2}$-stable. Consequently, the following mappings are all $L_{2}$-stable:

$$
\left[\begin{array}{l}
\dot{y}^{*} \\
\dot{w}
\end{array}\right] \rightarrow \dot{u}^{*},\left[\begin{array}{l}
\dot{y}^{*} \\
\dot{w}
\end{array}\right] \rightarrow \dot{v},\left[\begin{array}{l}
\dot{y}^{*} \\
\dot{w}
\end{array}\right] \rightarrow \dot{u},\left[\begin{array}{l}
\dot{y}^{*} \\
\dot{w}
\end{array}\right] \rightarrow \dot{y},\left[\begin{array}{l}
\dot{y}^{*} \\
\dot{w}
\end{array}\right] \rightarrow \dot{e}_{y}
$$

Proof. See Appendix A.

Proposition 2 (Saturation error analysis). Consider the control system composed of the system (1)-(2), subject to the saturation constraint (3), in closed-loop with the controller (12a)-(12b) where the polynomial $C(s)$ in (10) satisfies (15). Then, the mapping $\left[\dot{y}^{*} \dot{w} u^{*}-\operatorname{sat}\left(u^{*}\right)\right] \rightarrow v-u$ is $L_{2}$-stable.

Proof. See Appendix B.

The main result is now stated.

Theorem 1 ( $L_{2}$-tracking performance achievement). Consider the closed-loop control system described in Proposition 2. Then, the performance operator $(6 \mathrm{a}), \quad\left[\dot{y}^{*} \dot{w} u^{*}-\operatorname{sat}\left(u^{*}\right)\right] \rightarrow e_{y}$, is

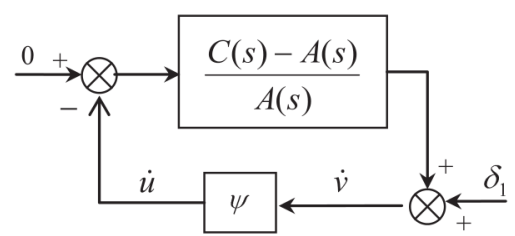

Fig. 1. Nonlinear feedback relating the signals $\dot{v}$ and $\dot{u}$ to the inputs' derivatives $\dot{y}^{*}$ and $\dot{w}$. 
actually $L_{2}$-stable. Consequently, the controller (12a)-(12b) features the $L_{2}$-tracking performance described by (7a)-(7c).

Proof. Operating $s R A$ on both sides of (4) gives, using (1):

$R A s \hat{e}_{y}(s)=R B e^{-s \tau} \hat{s}(s)-R A s \hat{y}^{*}(s)$

Similarly, operating $\Lambda B e^{-s \tau}$ on both sides of (12a) yields:

$\Lambda B e^{-s \tau} \hat{v}(s)=\Lambda B e^{-s \tau} \hat{u}(s)-R B e^{-s \tau} s \hat{u}(s)-B P e^{-s \tau} \hat{e}_{y}(s)$

Adding (16) and (17) yields, using (10):

$\Lambda C \hat{e}_{y}(s)=\Lambda B e^{-s \tau}(\hat{u}(s)-\hat{v}(s))+R A\left(s \hat{w}(s)-s \hat{y}^{*}(s)\right)$

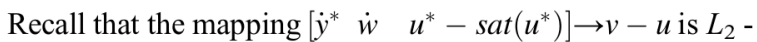
stable (by Proposition 2). Also, both $B e^{-s \tau} / C$ and $R A / \Lambda C$ are $L_{2}-$ stable. Then, one immediately gets from (18) that the mapping

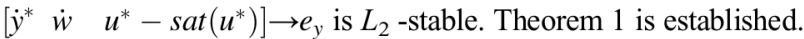
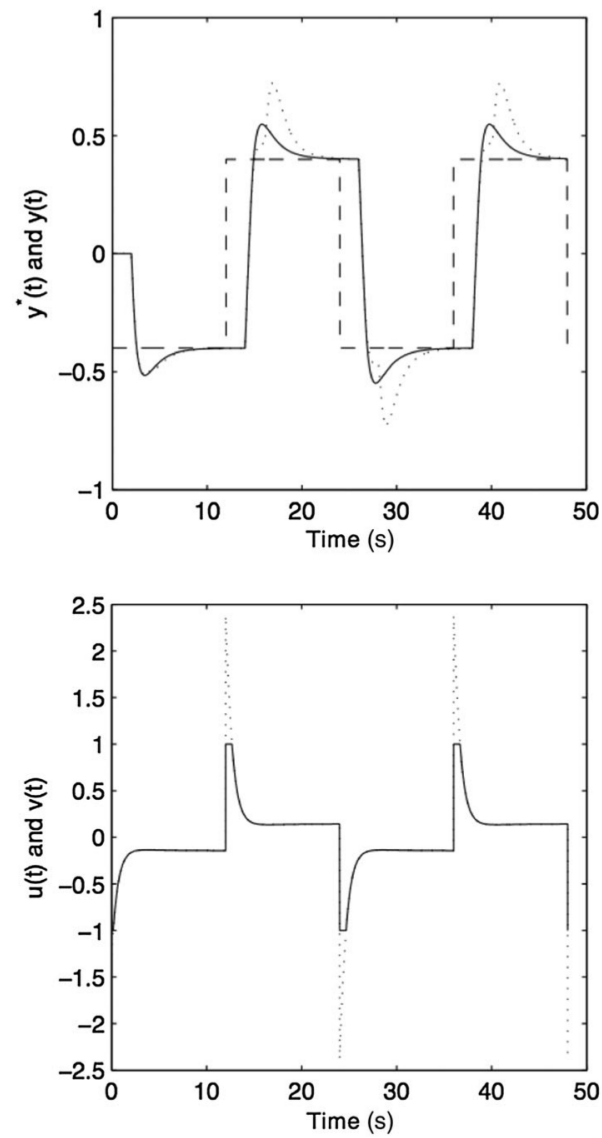

Fig. 2. Tracking performances of the saturated controller (12a-b), in presence of a constraint-compatible reference, when condition (15) is satisfied. Top: the system output $y(t)$ (solid), reference signal $y^{*}(t)$ (dashed). For comparison purpose, the system output $y(t)$ obtained with the compensator (20) is also shown (dotted). Bottom: the computed control $v(t)$ (dashed) and applied control $u(t)$ (solid).

\section{SIMULATION}

The system (1)-(2) is simulated with MATLAB/SIMULINK using the following numerical values:

$$
\begin{aligned}
A(s) & =s^{2}+1.25 s+0.25, B(s)=s+0.7, \\
\tau & =2 s, u_{M}=1
\end{aligned}
$$

Applying the control design of Section III to the example (19), a controller like (12a)-(12b) is obtained by solving the Bezout equation (10) using the following Hurwitz polynomials:

$C(s)=s^{2}+1.85 s+0.71, \quad \Lambda(s)=s^{2}+2.25 s+1.125$

It is readily checked that condition (15) is satisfied. No condition is imposed on $\Lambda(s)$. Solving equation (10) one gets $P(s)=3.13 s^{2}+4.26 s+1.12$ and $R(s)=s+2.85-3.16 e^{-s \tau}+0.233\left(\frac{1-e^{-(s+1) \tau}}{s+1}\right)-1.03\left(\frac{1-e^{-(s+0.25) \tau}}{s+0.25}\right)+$ $3.20\left(\frac{1-e^{-s t}}{s}\right)$.

Fig. 2 shows that the tracking performance is quite satisfactory. To better appreciate the performance of (12a)-(12b),
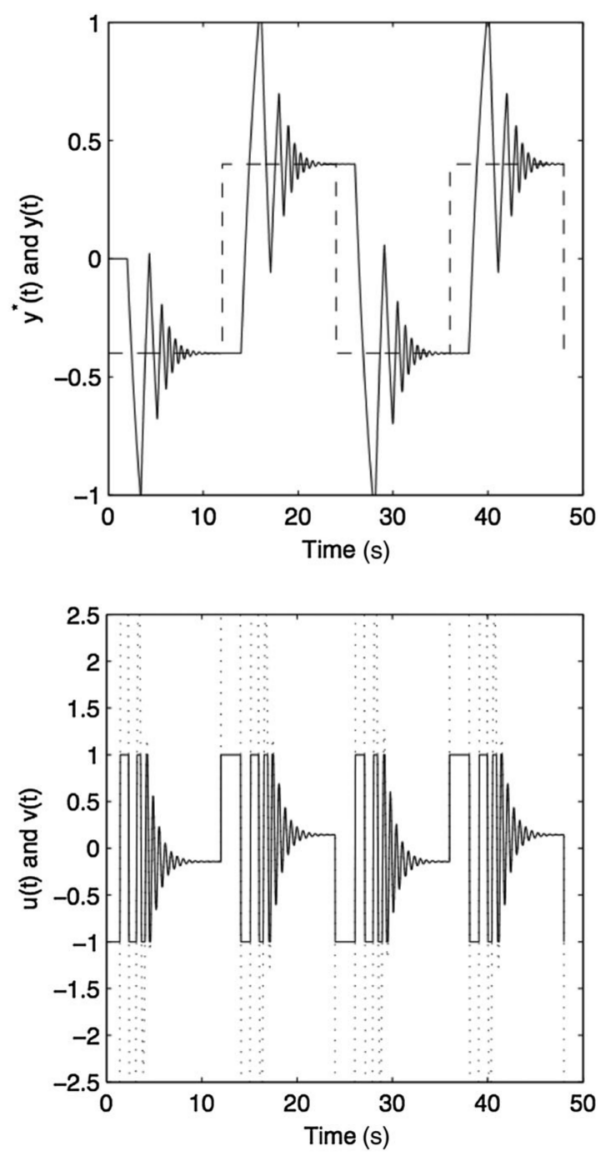

Fig. 3. Tracking performances of the saturated controller (12a-b), in presence of a constraint-compatible reference, when condition (15) is not satisfied. Top: the system output $y(t)$ (solid) and reference signal $y^{*}(t)$ (dashed). Bottom: the computed control $v(t)$ (dashed) and the applied control $u(t)$ (solid). 
the following finite spectrum assignment compensator, not conveniently accounting for saturation, is considered:

$$
\hat{v}(s)=\frac{P(s)}{s R(s)}\left(\hat{y}^{*}(s)-\hat{y}(s)\right) ; \quad u(t)=\operatorname{sat}(v(t))
$$

The corresponding tracking performances are illustrated by Fig. 2. Clearly, controller (12a)-(12b) performs much better than (20). To check the importance of condition (15), let us now take $C(s)=s^{2}+2 s+100$ and keep all remaining controller parameters unchanged. It is easily checked that condition (15) is no longer satisfied. The new closed-loop system responses are plotted in Fig. 3 which shows a clear deterioration of tracking performance.

\section{CONCLUSION}

Controlling input-delayed nonminimum-phase linear systems (1)-(2) is considered in the presence of actuator saturation (3) and output disturbance. The control design is performed within the ring of pseudo-polynomials, using (finite-spectrum) pole-placement design. It is formally shown that the controller (12a)-(12b) enjoys the appealing $L_{2} / L_{2 a}$ tracking features described by $(7 a)-(7 c)$. This result is quite powerful as it holds whatever the system inputs (reference, disturbance) being constraint-compatible or not.

\section{REFERENCES}

1. Lu, G., and D. W. C. Ho, "Generalized quadratic stabilization for discrete-time singular systems with time-delay and nonlinear perturbation," Asian J. Control, Vol. 7, No. 3, pp. 211-222 (2005).

2. Li, P., and J. Lam, "Synchronization in networks of genetics oscillators with delayed coupling," Asian J. Control, Vol. 13, No. 5, pp. 713-725 (2011).

3. Zaccarian, L., D. Nešic, and A. R. Teel, "L2 antiwindup for linear dead-time systems," Syst. Control Lett., Vol. 54, pp. 1205-1217 (2005).

4. Ahmed, A., M. U. Khan, M. Rehan, and N. Iqbal, "Two-Controller Anti-Windup Design for Enlarging Domain of Stability of Actuator Constrained StateDelay Systems," Asian J. Control, Vol. 15, No. 6, pp. 1821-1832 (2013).

5. Fu, Y. M., B. Zhou, and G. R. Duan, "Regional Stability and Stabilization of Time-Delay Systems with Actuator Saturation and Delay," Asian J. Control, Vol. 16, No. 3, pp. 845-855 (2013).

6. Zuo, Z., Y. Wang, and G. Zhang, "Stability analysis and controller design for linear time delay systems with actuator saturation," Amer. Control Conf., New York City, USA, pp. 5840-5844 (2007).

7. Zuo, Z., D. W. C. Ho, Y. Wang, and C. Yang, "A new approach for estimating the domain of attraction for linear systems with time-varying delay and saturating actuators," Asian Control Conf., Hong Kong, China, pp. 274-279 (2009).

8. Zhou, B., H. Gao, Z. Lin, and G. R. Duan, "Stabilization of linear systems with distributed input delay and input saturation," Automatica, Vol. 48, No. 5, pp. 712-724 (2012).

9. Van Assche, V., J. F. Lafay, and J. J. Loiseau, "Feedback realization of non-singular precompensators for linear systems over a polynomial ring," Int. J. Syst. Sci., Vol. 34, Issues $8 \& 9$, pp. 523-528 (2003).

10. Loiseau, J. J. "Algebraic tools for the control and stabilization of time-delay systems," Ann. Rev. Control, Vol. 24, pp. 135-149 (2000).

11. Khalil, H., Nonlinear Systems, Prentice Hall, Upper Saddle River, NJ, USA (2003).

12. Giri, F., F. Z. Chaoui, E. Chater, and D. Ghani, "SISO linear system control via saturating actuator: L2 tracking performance in presence of arbitrary shape inputs," Int. J. Control, Vol. 85, Issue 11, pp. 1694-1707 (2012).

13. Giri, F., V. Van Assche, A. Mahjoub, and F. Z. Chaoui, "Output reference tracking of input-delayed systems in presence of actuator saturation," IFAC Symp. Syst. Struct. Control, Ancona, Italy, pp. 261-266 (2010).

\section{APPENDIX A}

\subsection{Proof of Proposition 1}

Let us show that the mapping $\psi$, in Fig. 1, belongs to the

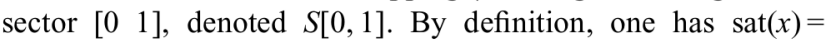
$\operatorname{sgn}(x) \min \left(|x|, u_{M}\right)$. Then, it is readily seen that:

$0 \leq \frac{d \text { sat }}{d x}(x) \leq 1, \quad \forall x \in \mathbf{R}$

On the other hand, one gets from $(12 b)$ :

$$
\dot{u}(t)=\frac{d \text { sat }}{d v}(v(t)) \times \dot{v}(t)
$$

Multiplying both sides of (A2) by $\dot{v}(t)$ gives, $\dot{v} \times \dot{u}=$ $\frac{d \text { sat }}{\mathrm{d} v}(v) \dot{v}^{2}$. This, together with (A1), implies that $0 \leq \dot{v} \times \dot{u} \leq$ $(\dot{v})^{2}$ which proves that $\psi \in S[0,1]$. Then, it follows applying the circle criterion [11] that the feedback of Fig. 1 is $L_{2}$-stable provided that $\inf _{0 \leq \omega<+\infty} \operatorname{Re}((C(j \omega)-A(j \omega)) / A(j \omega))>-1$ which is nothing other than (15). Then, it follows from Fig. 1 that the two mappings $\delta_{1} \rightarrow \dot{v}$ and $\delta_{1} \rightarrow \dot{u}$ are $L_{2}$-stable. As $S(s) / \Lambda(s)$ is asymptotically stable, one has from (14) that, the mapping $\left[\begin{array}{ll}\dot{y}^{*} & \dot{w}\end{array}\right] \rightarrow \delta_{1}$ is also $L_{2}$-stable. Combining the above results, one gets that $\left[\begin{array}{ll}\dot{y}^{*} & \dot{w}\end{array}\right] \rightarrow \dot{v}$ and $\left[\dot{y}^{*} \quad \dot{w}\right] \rightarrow \dot{u}$ are both $L_{2}$-stable. The rest of the proof follows similar arguments. 


\section{APPENDIX B}

\subsection{Proof of Proposition 2}

Using (4), one gets from (12a):

$$
\begin{aligned}
\hat{v}(s)-\hat{u}(s) & =-\frac{R}{\Lambda} s \hat{u}(s)-\frac{P}{\Lambda} \hat{e}_{y}(s) \\
& =\frac{p_{0} b_{0}}{\lambda_{0} a_{0}}\left(\hat{u}^{*}(s)-\hat{u}(s)\right)+\hat{\delta}_{2}(s)+\hat{\delta}_{3}(s)+\hat{\delta}_{4}(s)
\end{aligned}
$$

with $\quad \hat{\delta}_{2}(s)=\left(\frac{b_{0}}{a_{0}}-\frac{B}{A}\right) \frac{P e^{-s t}}{\Lambda} \hat{u}^{*}(s)+\frac{P}{\Lambda} \hat{w}(s), \hat{\delta}_{3}(s)=-\frac{R}{\Lambda} s \hat{u}(s)-$ $\frac{\left(P-p_{0}\right) B e^{-\tau s}}{s \Lambda A}\left(s \hat{u}(s)-s \hat{u}^{*}(s)\right), \quad \hat{\delta}_{4}=\left(\frac{s_{0} B e^{-s t}}{\Lambda A}-\frac{s_{0} b_{0}}{\lambda_{0} a_{0}}\right)\left(\hat{u}^{*}(s)-\hat{u}(s)\right)$. In the time domain, one gets:

$$
v(t)-u(t)=\frac{c_{0}}{a_{0}}\left(\operatorname{sat}\left(u^{*}(t)\right)-u(t)\right)+\delta_{6}(t)
$$

with $\delta_{5}=\frac{c_{0}}{a_{0}}\left(\operatorname{sat}\left(u^{*}\right)-u^{*}\right)$ and $\delta_{6}=\delta_{2}+\delta_{3}+\delta_{4}+\delta_{5}$, where we have used the fact that $p_{0}=P(0)$ and $p_{0} b_{0}=\lambda_{0} c_{0}$ (obtained letting $s=0$ in (10)). One can demonstrate that all mappings $\mu \rightarrow \delta_{i}$ $(i=2 \ldots 5)$ are $L_{2}$-stable where:

$$
\mu \stackrel{\operatorname{def}}{=}\left[\dot{y}^{*} \dot{w} u^{*}-\operatorname{sat}\left(u^{*}\right)\right]
$$

The detailed proof is presently provided for the mapping $\mu \rightarrow \delta_{2}$. The result for the remaining mappings follows similarly. By definition one has:

$$
\hat{\delta}_{2}(s)=\left(\frac{b_{0}}{a_{0}}-\frac{B}{A}\right) \frac{P e^{-s \tau}}{\Lambda} \hat{u}^{*}(s)=\frac{D(s)}{a_{0} A(s)} \frac{P e^{-s \tau}}{\Lambda} s \hat{u}^{*}(s)
$$

with $D(s)=b_{0}\left(s^{n-1}+a_{n-1} s^{n-2}+\ldots+a_{1}\right)-a_{0}\left(b_{n-1} s^{n-2}+\ldots+\right.$ $\left.b_{1}\right)$, where we have used the fact that:

$$
\begin{aligned}
b_{0}\left(s^{n}\right. & \left.+a_{n-1} s^{n-1}+\ldots+a_{1} s+a_{0}\right) \\
& -a_{0}\left(b_{n-1} s^{n-1}+\ldots+b_{1} s+b_{0}\right)=D(s) \times s
\end{aligned}
$$

In view of (2), it is checked that $D(s) / a_{0} A(s)$ is strictly proper and asymptotically stable (because $A(s)$ is Hurwitz). Similarly, it is checked that $P e^{-s \tau} / \Lambda$ is asymptotically stable. Then, it follows from (B3) that the mapping $\dot{u}^{*} \rightarrow \delta_{2}$ is $L_{2}$-stable. We know by Proposition 1 that the mapping $\left[\dot{y}^{*} \quad \dot{w}\right]^{T} \rightarrow \dot{u}^{*}$ is $L_{2}$-stable. Then, one gets that $\mu \rightarrow \delta_{2}$ is $L_{2}$-stable. The rest of the proof consists in showing that:

$$
|v(t)-u(t)| \leq\left|\delta_{6}(t)\right|, \text { for all } t
$$

It is clear that (B4) holds when $|v(t)| \leq u_{M}$ because one then has $v(t)-u(t)=0$, due to $(12 \mathrm{~b})$. So let us prove that (B4) holds in the case where $|v(t)|>u_{M}$. As $A$ and $C$ are Hurwitz, their coefficients $a_{0}$ and $c_{0}$ are positive (by RouthHurwitz criterion). Furthermore, as $|v(t)|>u_{M}$, one has from (12b) that $|u(t)|=u_{M} \operatorname{sgn}(v(t))$ which implies that $|u(t)|>\left|\operatorname{sat}\left(u^{*}(t)\right)\right|$. Then, multiplying both sides of (B1) by $\operatorname{sgn}(v(t))$ and using the fact that $\operatorname{sgn}(v(t))=\operatorname{sgn}(u(t))$, one gets for all $t$ :

$$
\begin{gathered}
|v(t)-u(t)|=\frac{c_{0}}{a_{0}}\left(\operatorname{sat}\left(u^{*}(t)\right) \operatorname{sgn}(v(t))-|u(t)|\right) \\
+\delta_{6}(t) \operatorname{sgn}(v(t)) \leq \delta_{6}(t) \operatorname{sgn}(v(t))
\end{gathered}
$$

This implies, on one hand, that $\delta_{6}(t) \operatorname{sgn}(v(t))$ is nonnegative and, on the other hand, that $|v(t)-u(t)| \leq\left|\delta_{6}(t)\right|$. Hence, inequality (B4) does hold which proves Proposition 2. 